\title{
The influence of molecular architecture on the macroscopic lubrication properties of the brush-like co-polyelectrolyte poly(L-lysine)-g-poly(ethylene glycol) (PLL-g-PEG) adsorbed on oxide surfaces
}

\author{
Markus Müller ${ }^{\mathrm{a}}$, Seunghwan Lee ${ }^{\mathrm{a}}$, Hugh A. Spikes ${ }^{\mathrm{b}}$ and Nicholas D. Spencer ${ }^{\mathrm{a}, *}$ \\ ${ }^{a}$ Laboratory for Surface Science and Technology, Department of Materials, ETH-Zürich, Sonneggstrasse 5, Zürich, CH-8092, Switzerland \\ ${ }^{b}$ Tribology Section, Department of Mechanical Engineering, Imperial College, London, SW7 2BX UK
}

Received 21 February 2003; accepted 29 May 2003

\begin{abstract}
The co-polymer poly(L-lysine)-g-poly(ethylene glycol) (PLL-g-PEG) has been investigated as a potential biomimetic boundarylubrication additive for aqueous lubrication systems. In this work, the influence of the co-polymer's architecture on its tribological performance has been investigated. The architectural parameters investigated comprise side-chain (PEG) length, Lys/PEG grafting ratio and backbone chain (PLL) length. The tribological approaches applied in this work include ultra-thin-film interferometry, the mini-traction machine (MTM), and pin-on-disk tribometry. Both an increase in the molecular weight of the PEG side chains and a reduction in the grafting ratio result in an improvement in the lubricating properties of aqueous PLL- $g$-PEG solution at low speeds. MTM measurements show that an increase in the molecular weight of the PLL backbone results in an increase of the coefficient of friction.
\end{abstract}

KEY WORDS: poly(L-lysine)-g-poly(ethylene glycol) (PLL-g-PEG), aqueous lubrication, polymer architecture, boundary lubrication

\section{Introduction}

In a previous paper [1] we explored the application of the poly(L-lysine)-g-poly(ethylene glycol) (PLL-g-PEG) as a potential biomimetic boundary-lubrication additive for aqueous lubrication systems. We showed that, for oxide tribo-systems, the boundary-lubrication properties of aqueous buffer solution are significantly improved upon addition of PLL- $g$-PEG. On the molecular scale, Yan et al. [2] recently investigated the influence of the architectural parameters of PLL-g-PEG on its lubrication properties in aqueous solution by means of atomic force microscopy (AFM). The results revealed that the adsorption of PLL- $g$-PEG onto silicon oxide surfaces significantly reduces friction, with the lowest friction being observed when polymer adsorption occurs on both surfaces. As shown in figure 1, PLL-gPEG is composed of a polyionic PLL backbone with covalently bound PEG side chains. The enhanced lubricating effect of PLL- $g$-PEG in aqueous solution was ascribed to its unique adsorption behavior: the positively charged amino groups of the PLL backbone are electrostatically attracted to negatively charged oxide surfaces, forcing the PEG side chains into a dense, brush-like structure. Polymer "brushes", i.e., systems of polymer chains end-grafted onto surfaces, are

\footnotetext{
* To whom correspondence should be addressed. E-mail: nicholas. spencer@mat.ethz.ch
}

often used to modify surface forces and have important technological applications in many areas, including colloidal stabilization and adherence [3,4]. Over the past ten years, attention has been paid to end-grafted polymers that exhibit unique tribological properties in "good" solvents, and it is suggested that brush-like polymer layers may be useful as lubricants. Models for the shear interactions between polymer-bearing surfaces have been described by both experimental [5-9] and computer-simulation studies [10,11]. The origin of the low frictional forces between brush-bearing surfaces has been attributed both to steric repulsion between the polymers supporting high normal loads and to intermolecular interactions between the polymer brushes and the solvent molecules which maintain a lubricating fluid layer at the sheared interfacial region [11]. Extremely low coefficients of friction (COF) (as low as the detection limit $<0.001$ ) have been reported in nanotribological studies performed by means of the surface forces apparatus (SFA) [7].

Most tribological studies concerned with investigating the lubrication properties of end-grafted polymers have been performed at the nanometer scale in a verylow-load regime. However, little is known about the lubrication properties of end-grafted polymers on a macroscopic scale, which is more relevant when considering technical and engineering applications such as polymer-bearing interfaces. In our previous work [1], a significant reduction of the macroscopic 


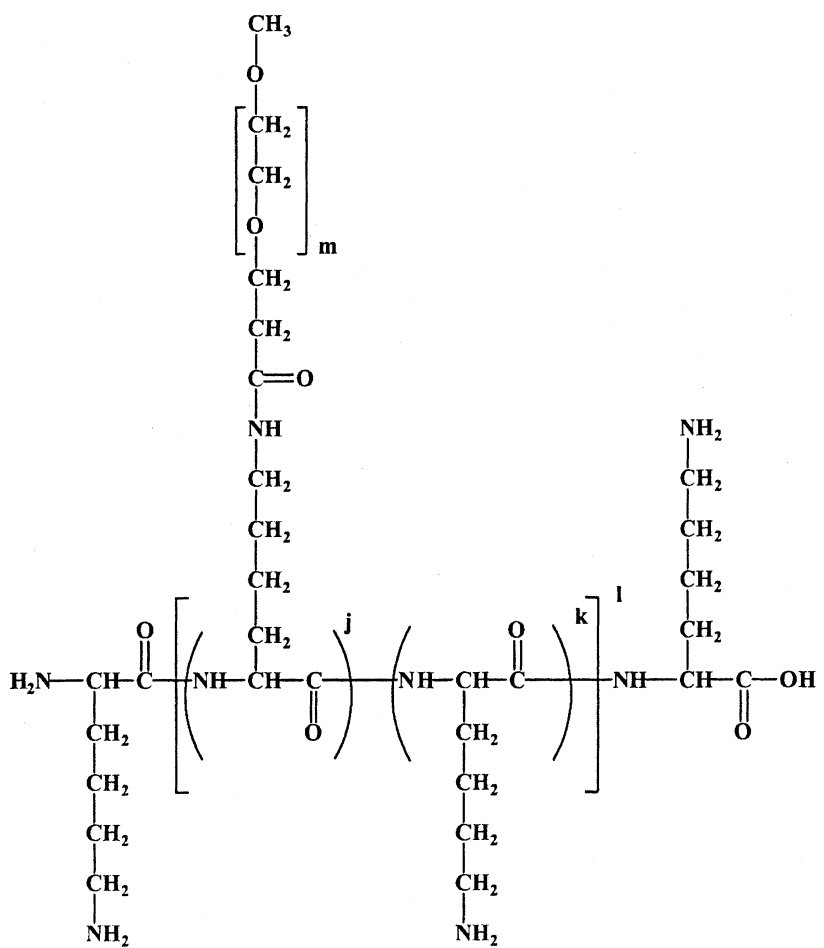

Figure 1. A schematic illustration of the chemical structure of poly(L-lysine)-g-poly(ethylene glycol) (PLL-g-PEG).

COF for oxide-based tribo-systems was observed when adding PLL-g-PEG to aqueous buffer solution. The present study is a continuation of this work, focusing on the architectural parameters of the PLL- $g$-PEG copolymer and its influence on the macroscopic lubrication properties. The architectural parameters investigated in this work include side-chain (PEG) length, Lys/ PEG grafting ratio and backbone (PLL) length. The experimental techniques employed are ultra-thin-film interferometry, the mini-traction machine (MTM), and pin-on-disk tribometry.

\section{Materials and methods}

\subsection{Synthesis of poly (L-lysine) grafted poly(ethylene glycol)}

The following abbreviations are used for the various polymers discussed in this paper, to indicate the average molecular weight and the grafting ratio: PLL(x)-g[y]PEG(z) signifies that the graft co-polymer has a PLL backbone of mol. wt $\mathrm{x} \mathrm{kDa,} \mathrm{a} \mathrm{graft} \mathrm{ratio,} \mathrm{y}$, of lysine unit/PEG side chain, and PEG side chains of mol. wt $\mathrm{z}$ kDa.

Table 1 shows the details of the reagent masses that were used to synthesize the different polymers according to a method described in previous publications [12]. Briefly, poly(L-lysine) hydrobromide (Fluka, Switzerland) was dissolved at a concentration of $100 \mathrm{mM}$ in SBB (sodium borate buffer solution, $50 \mathrm{mM}$ ) adjusted to $\mathrm{pH}$ 8.5. The solution was filter sterilized $(0.22 \mu \mathrm{m}$ pore-
Table 1.

Details of the masses that were used to synthesize the polymers possessing different architecture. PLL(x)-g[y]-PEG(z) signifies that the graft copolymer has a PLL backbone of mol. wt x kDa; a graft ratio, y, of lysine unit/PEG side chain, and PEG side chains of mol. wt $\mathrm{z}$ kDa.

\begin{tabular}{lcc}
\hline $\begin{array}{l}\text { Polymer } \\
\text { PLL(x)-g[y]-PEG(z) }\end{array}$ & PLL & PEG \\
\hline PLL(20)-g[3.4]-PEG(2) & $245.0 \mathrm{mg}$ & $689.6 \mathrm{mg}$ \\
PLL(20)-g[3.4]-PEG(5) & $106.9 \mathrm{mg}$ & $751.9 \mathrm{mg}$ \\
PLL(20)-g[2.2]-PEG(2) & $107.5 \mathrm{mg}$ & $543.9 \mathrm{mg}$ \\
PLL(20)-g[5.7]-PEG(2) & $278.2 \mathrm{mg}$ & $433.2 \mathrm{mg}$ \\
PLL(350)-g[3.4]-PEG(2) & $106.9 \mathrm{mg}$ & $751.9 \mathrm{mg}$ \\
\hline
\end{tabular}

size filter). For the grafting of PEG onto PLL, the $N$-hydroxysuccinimidyl ester of methoxypoly (ethylene glycol) propionic acid (mPEG-SPA, Nektar, Huntsville, AL, USA) was added to PLL-HBr solution. The reaction was allowed to proceed for $6 \mathrm{~h}$ at room temperature, after which the reaction mixture was dialyzed (Spectra-Por, mol. wt cutoff size $6-8 \mathrm{kDa}$, Spectrum, Houston, TX, USA) for $48 \mathrm{~h}$ against deionized water. The product was freeze-dried and stored in powder form at $-20^{\circ} \mathrm{C}$. Detailed analytical information concerning the product produced by this method is available in previous publications $[12,13]$.

For all subsequent experiments, PLL(x)- $g[\mathrm{y}]-\mathrm{PEG}(\mathrm{z})$ was dissolved at a concentration of $0.25 \mathrm{mg} / \mathrm{ml}$ in $10 \mathrm{mM}$ HEPES (4-(2-hydroxyethyl)piperazine-1-ethanesulfonic acid (Fluka, Switzerland), adjusted to $\mathrm{pH} 7.4$ with $1.0 \mathrm{M} \mathrm{NaOH}$ solution).

\subsection{Ultra-thin-film interferometry}

The lubricant film-forming ability of the test solutions in a high-pressure rolling contact was measured by means of ultra-thin-film interferometry. The detailed method has been described elsewhere [1,14]. A lubricated contact is formed between a loaded stainless steel ball (radius $R=9.5 \mathrm{~mm}$, AISI 440, RMS surface roughness $11 \mathrm{~nm}$, PCS Instruments, London, UK) and the flat surface of a silicate glass disk (RMS surface roughness $2 \mathrm{~nm}$ ). The disk drives the ball in nominally pure rolling and although the precise motion of the ball is not externally controlled, it is believed to roll against the disk with less than $2 \%$ sliding at speeds up to $1 \mathrm{~m} / \mathrm{s}$. The surface of the glass disk in contact with the ball is coated with a very thin, semi-reflecting layer of chromium, on top of which is a 400-nm layer of transparent silica which acts as a spacer layer. Thus the tribological pair consists of $\mathrm{FeO}_{x} / \mathrm{SiO}_{x}$. White light is shone through the glass into the contact, where a proportion is reflected back from the chromium layer while the remainder passes through the silica layer and the polymer-containing water before being reflected from the steel ball surface. Since the two reflected beams 
have traveled different distances, they interfere with a relative phase shift that depends on the path difference, i.e., on the sum of silica and lubricant film thickness. The interference pattern from the contact is passed to a spectrometer and the resultant dispersed light is analyzed to determine the precise wavelength of maximum constructive interference from the centre of the contact. This yields an accurate measure of the composite film thickness, from which the thickness of the spacer layer can be subtracted. For the calculation of the film thickness, the refractive index of the lubricant has to be known. Based on our previous work [1], a uniform refractive index of 1.4 has been used for the calculations of the film thickness for all PLL- $g$-PEG architectures. All measurements were carried out at room temperature $\sim 25^{\circ} \mathrm{C}$ and a fixed load of $10 \mathrm{~N}$, which corresponds to a maximum Hertzian contact pressure of $0.41 \mathrm{GPa}$. The maximum rotation speed of the disk track was limited to $1 \mathrm{~m} / \mathrm{s}$. Each measurement was performed on a fresh disk track by varying the radii of measurement within 35 to $42 \mathrm{~mm}$ and a fresh stainless steel ball was used for each test.

\subsection{Mini-traction machine (MTM)}

A mini-traction machine (MTM, PCS Instruments, London, UK) was employed to characterize the lubrication properties of the polymer solutions in a mixed sliding/rolling contact regime. The set-up of the instrument is described in ref. [1]. A lubricated contact is formed between a polished stainless steel ball (radius $R=9.5 \mathrm{~mm}$, AISI 440, RMS surface roughness $11 \mathrm{~nm}$, PCS Instruments, London, UK) and a flat silicate glass disk (RMS surface roughness $2 \mathrm{~nm}$, PCS Instruments, London, UK), thus representing an $\mathrm{FeO}_{x} / \mathrm{SiO}_{x}$ interface. The MTM provides a mixed sliding/rolling contact through the independent control of the ball and disk velocities. The slide/roll ratio, SRR, is defined as the percentage ratio of the difference and the mean of the ball speed $\left(u_{\text {ball }}\right)$ and disk speed $\left(u_{\text {disk }}\right)$, i.e., $\operatorname{SRR}=\left[\left|u_{\text {ball }}-u_{\text {disk }}\right| /\left(u_{\text {ball }}+u_{\text {disk }}\right) / 2\right] \times 100 \%$. Thus, $\mathrm{SRR}=0 \%$ (i.e. $\left.u_{\text {ball }}=u_{\text {disk }}\right)$ represents a pure rolling contact and higher SRR values represent a higher portion of the sliding character. With the software provided (PCS Instruments, MTM version 1.0, London, $\mathrm{UK}$ ), values in the range $\mathrm{SRR}=1$ to $200 \%$ were accessible. At a given SRR, the COF $(\mu=F / N)$ is measured as a function of mean speed. For the current work, MTM tests were initially conducted at several different SRR values between 5 to $25 \%$ but no dependence of friction on SRR in this range was detected. Therefore a standard SRR value of $10 \%$ was chosen, which was large enough to provide reliable friction measurement but sufficiently small so as to be comparable with the nominally pure rolling conditions of the ultra-thin-film interferometry work. It has been shown that fluid entrainment and thus film thickness is independent of slide/roll ratio within the range $\mathrm{SRR}=0$ to $50 \%$ [15]. MTM tests on polymer solutions were performed at a fixed load of $10 \mathrm{~N}$ and a controlled temperature of $25^{\circ} \mathrm{C}$, the same conditions as in the ultra-thin-film interferometry. The disk track radius was fixed at $20.7 \mathrm{~mm}$ and for each measurement, a new stainless steel ball and a new glass disk were used.

\subsection{Pin-on-disk tribometry}

A pin-on-disk tribometer (CSEM, Neuchâtel, Switzerland) was employed in this work to characterize the influence of the architectural parameters of PLL- $g$-PEG on lubrication properties in pure sliding conditions as a function of load. There were two reasons for using pinon-disk tribometry for pure sliding tests, rather than the MTM. One is the availability of a wide range of substrate materials (e.g., ceramics) for pin-on-disk, which was important in determining the ideal settings and material pairings at the beginning of the experimental work. More importantly, the pin-on-disk was able to operate at slower sliding speeds than the MTM and thus ensures full boundary-lubrication conditions, as discussed later in this paper. In the pin-on-disk setup, a fixed, spherical steel pin $(6 \mathrm{~mm}$ in diameter, DIN 5401-20 G20, Hydrel AG, Romanshorn, Switzerland) was loaded against a flat glass disk (RMS surface roughness $2 \mathrm{~nm}$ ). The load was controlled by placing dead weights on top of the pin holder ( 1 to $5 \mathrm{~N}$ ) and the friction forces were measured by a strain gauge at a constant speed of $0.005 \mathrm{~m} / \mathrm{s}$ at room temperature $\sim 25^{\circ} \mathrm{C}$. The maximum Hertzian contact pressure in this configuration is calculated to be $0.41,0.51$, and $0.70 \mathrm{GPa}$ at applied loads of 1,2 , and $5 \mathrm{~N}$, respectively. The number of rotations of the disk was limited to 100 . For each friction measurement, a new steel ball and a fresh track on the glass disk was used. The friction signals were recorded with a Macintosh Power PC using Labview and an ADC card of the MIO family (both from National Instruments, Austin, TX, USA).

\subsection{Cleaning of the tribo-pairs}

All the instrumental parts that are expected to be in contact with the polymer solution, including the balls and disks of all tribological instruments, were oxygenplasma cleaned for ca. $2 \mathrm{~min}$ in a Harrick Plasma Cleaner/Sterilizer PDC-32G instrument (Ossining, NY, USA) prior to the measurements. Before the plasma cleaning, the instrumental parts and the tribo-pair of ultra-thin-film interferometry and MTM were degreased by sonication in toluene for $10 \mathrm{~min}$, followed by sonication in isopropanol for $10 \mathrm{~min}$, and dried in nitrogen atmosphere. The pins, disks and assembly parts 


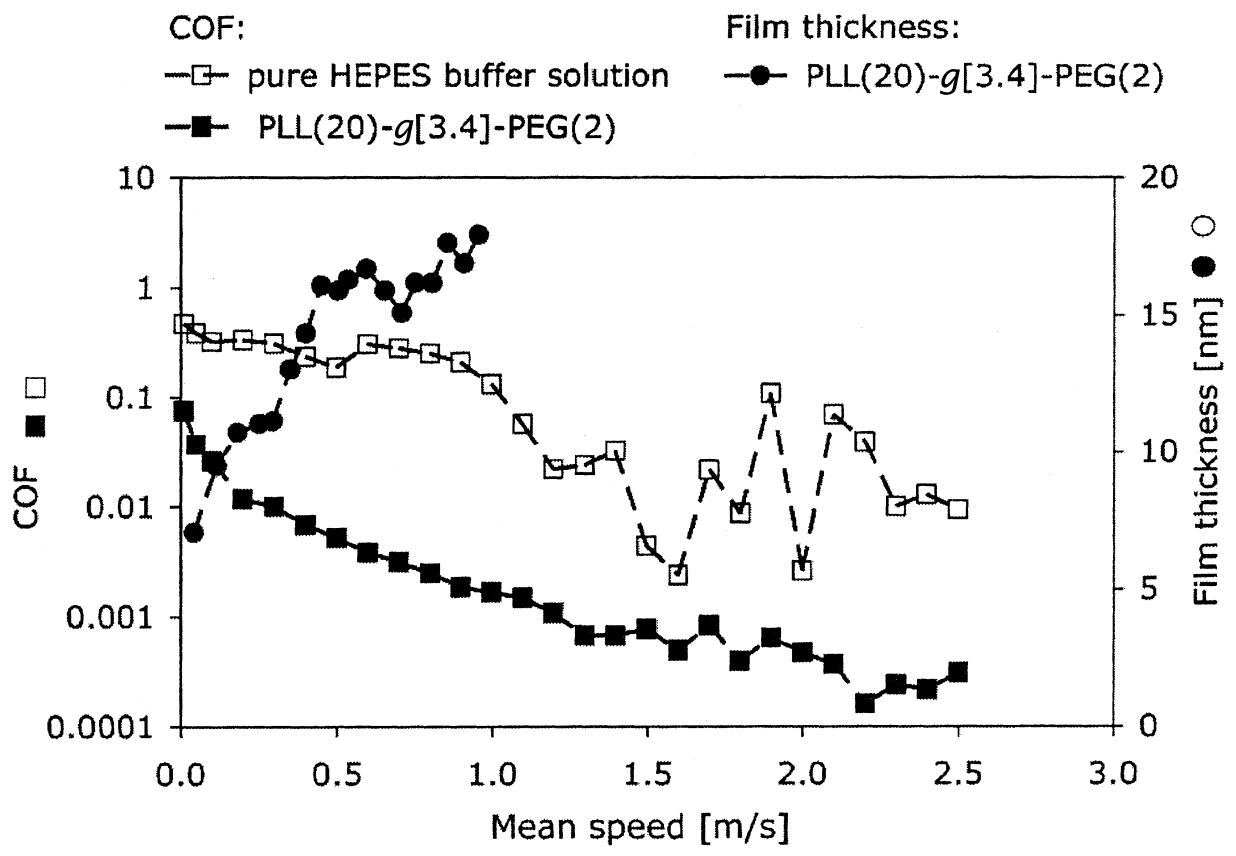

Figure 2. A direct comparison of the coefficient of friction (y-axis on the left-hand-side) and the lubricant film thickness (y-axis on the right-handside) for both polymer-free and PLL(20)-g[3.4]-PEG(2)-containing HEPES buffer solution, measured by MTM and ultra-thin-film interferometry. Squares are for the coefficient of friction and circles for the lubricant film thickness. The lines between data points serve as a guide for the eye. Ball $=$ stainless steel $(19 \mathrm{~mm}$ in diameter), substrate $=$ silica, buffer solution $=10 \mathrm{mM}$ HEPES ( $\mathrm{pH}$ 7.4), polymer concentration $=0.25 \mathrm{mg} / \mathrm{ml}, \operatorname{load}=10 \mathrm{~N}, \mathrm{~T}=25^{\circ} \mathrm{C}$.

of pin-on-disk were sonicated in ethanol for $10 \mathrm{~min}$ and dried in nitrogen atmosphere.

\section{Results}

\subsection{Lubrication properties of $P L L-\mathrm{g}-P E G$ in aqueous buffer solution compared with pure buffer solution}

Figure 2 compares the coefficient of friction (COF) of PLL(20)- $g$ [3.4]-PEG(2)-containing HEPES buffer solution and pure (polymer-free) HEPES buffer solution as a function of mean rolling speed, in a glass/steel tribopair measured using the MTM. Also shown in this figure is the film thickness of the polymer solution measured as a function of rolling speed using ultra-thinfilm interferometry. As shown in our previous work [1], the film thickness of pure HEPES buffer solution could not be measured reliably below $1 \mathrm{~m} / \mathrm{s}$ because of significant wear and resulting damage to the coatings on the glass disk. However, upon addition of PLL(20)$g$ [3.4]-PEG(2) to the pure HEPES buffer solution, no such damage occurred and film thickness was found to increase from 7 to $18 \mathrm{~nm}$ as the speed was increased from 0.01 to $1 \mathrm{~m} / \mathrm{s}$. At speeds above $1 \mathrm{~m} / \mathrm{s}$ an erratic and irreproducible decrease of the film thickness was seen, possibly due to damage to the disk coating. Hence, the speed range investigated by ultra-thin-film interferometry was limited to the range of 0.01 to $1 \mathrm{~m} / \mathrm{s}$.

The friction-speed curve obtained for pure HEPES buffer solution shows a constant COF of around 0.5 for a speed below $1 \mathrm{~m} / \mathrm{s}$. However, at higher speed, the COF became unstable and oscillated with a high irregular amplitude between 0.1 and 0.01. On the other hand, PLL(20)-g[3.4]-PEG(2) solution displayed a quite uniform decrease of the COF over the whole speed range, to reach values at least an order of magnitude lower than those obtained with pure HEPES buffer solution.

Figure 3 shows the results of pin-on-disk tribometry, also performed on a steel/glass tribo-pair, at a constant, pure sliding speed of $0.005 \mathrm{~m} / \mathrm{s}$ at three applied loads. For the PLL(20)-g[3.4]-PEG(2) solution, the COF $(\sim 0.15)$ was about half that of pure HEPES buffer solution $(\sim 0.3)$ at all loads investigated. No reduction of the COF was observed upon addition of free PEG of similar molecular weight to the pure HEPES solution (data not shown). The COF for all test fluids decreased with increasing the load from 1 to $2 \mathrm{~N}$, but remained broadly constant as the load was further increased to $5 \mathrm{~N}$.

\subsection{The influence of the PLL-g-PEG architecture on the tribological properties}

The influence of the architectural parameters of the PLL- $g$-PEG polymers on film formation and friction was investigated by varying the side chain (PEG) length, the Lys/PEG grafting ratio, and the backbone (PLL) length. 


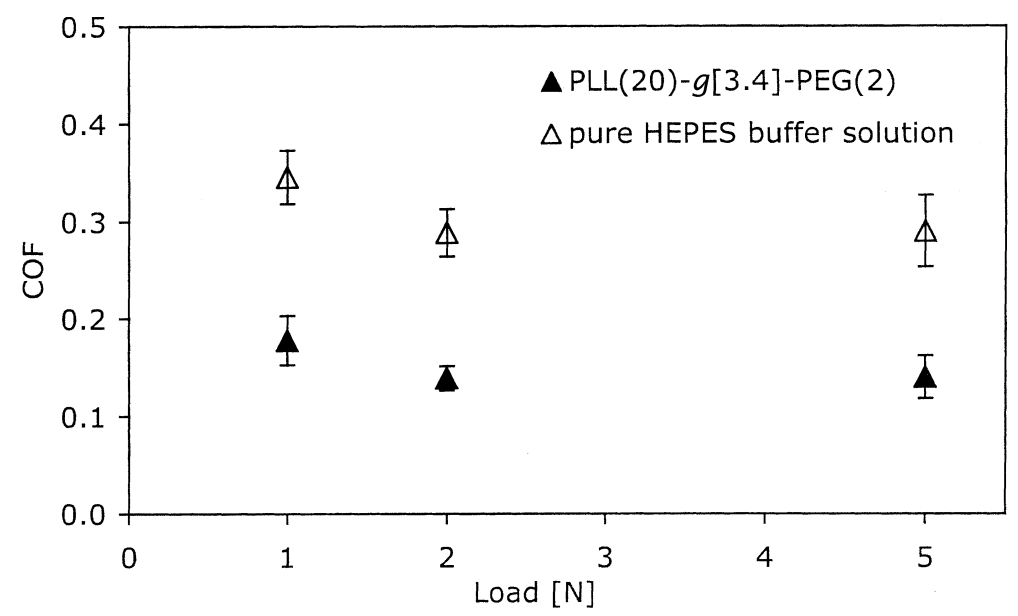

Figure 3. Coefficient of friction versus load determined by pin-on-disk tribometry for both polymer-free (white triangles) and PLL(20)- $g$ [3.4]PEG(2)-containing (black triangles) HEPES buffer solution. Mean values of three experiments \pm standard deviation. Ball $=$ steel $(6 \mathrm{~mm}$ in diameter), disk $=$ glass, buffer solution $10 \mathrm{mM}$ HEPES $(\mathrm{pH} 7.4)$, polymer concentration $=0.25 \mathrm{mg} / \mathrm{ml}$, load $=10 \mathrm{~N}, \mathrm{~T}=25^{\circ} \mathrm{C}$.

\subsubsection{Influence of the molecular weight of the PEG chain}

Two PLL-g-PEG polymers with different PEG sidechain molecular weights $\left(M_{w}=2 \mathrm{kDa}\right.$ and $\left.5 \mathrm{kDa}\right)$ but the same molecular weight of the PLL backbone $\left(M_{w}=20 \mathrm{kDa}\right)$ and the same Lys/PEG grafting ratio (3.4), were synthesized. Figure 4 shows the results of the MTM and ultra-thin-film interferometry experiments on solutions of these polymers. Over the entire range of speed $(0.01$ to $1 \mathrm{~m} / \mathrm{s})$, the polymers with $5 \mathrm{kDa}$ PEG side chains form thicker lubricant films than those with $2 \mathrm{kDa}$ chains. Under the same conditions, the COF is consistently lower for the polymer with $5 \mathrm{kDa}$ PEG side chains than for the $2 \mathrm{kDa}$ side-chain one. Figure 5 shows the COF measured as a function of load by means of pin-on-disk tribometry. Again, the polymer with the $5 \mathrm{kDa}$ side chain reveals a significantly reduced $\mathrm{COF}$ compared to the polymer with $2 \mathrm{kDa}$ chains.

\subsubsection{Influence of the Lys/PEG grafting ratio}

The influence of the Lys/PEG grafting ratio on frictional properties and interfacial film formation of PLL- $g$-PEG was investigated by varying grafting ratio while keeping molecular weight of the PLL backbone and PEG chain constant at $20 \mathrm{kDa}$ and $2 \mathrm{kDa}$, respectively. Grafting ratios of $g=2.2,3.4$, and 5.7 were investigated. Figure 6 shows the results of both

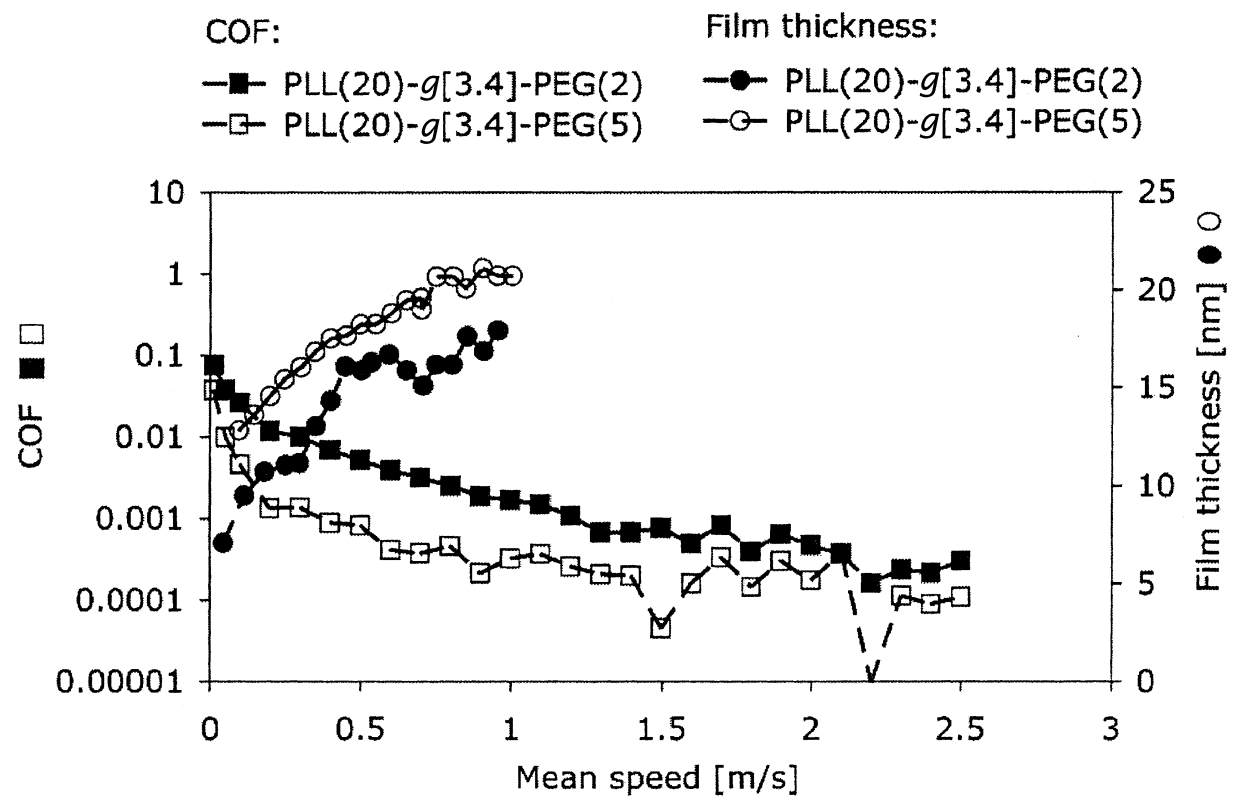

Figure 4. The influence of the molecular weight of the PEG side chains on the coefficient of friction (squares; y-axis on the left-hand-side) and lubricant film thickness (circles; y-axis on the right-hand-side) was measured as a function of speed by means of MTM and ultra-thin-film interferometry. The test lubricants were aqueous buffer solutions containing either PLL(20)-g[3.4]-PEG(2) (black symbols) or PLL(20)- $g$ [3.4]PEG(5) (white symbols). The lines between data points serve as a guide for the eye. Ball = stainless steel $(19 \mathrm{~mm}$ in diameter $)$, substrate $=$ silica, buffer solution $=10 \mathrm{mM}$ HEPES $(\mathrm{pH} 7.4)$, polymer concentration $=0.25 \mathrm{mg} / \mathrm{ml}$, load $=10 \mathrm{~N}, \mathrm{~T}=25^{\circ} \mathrm{C}$. 


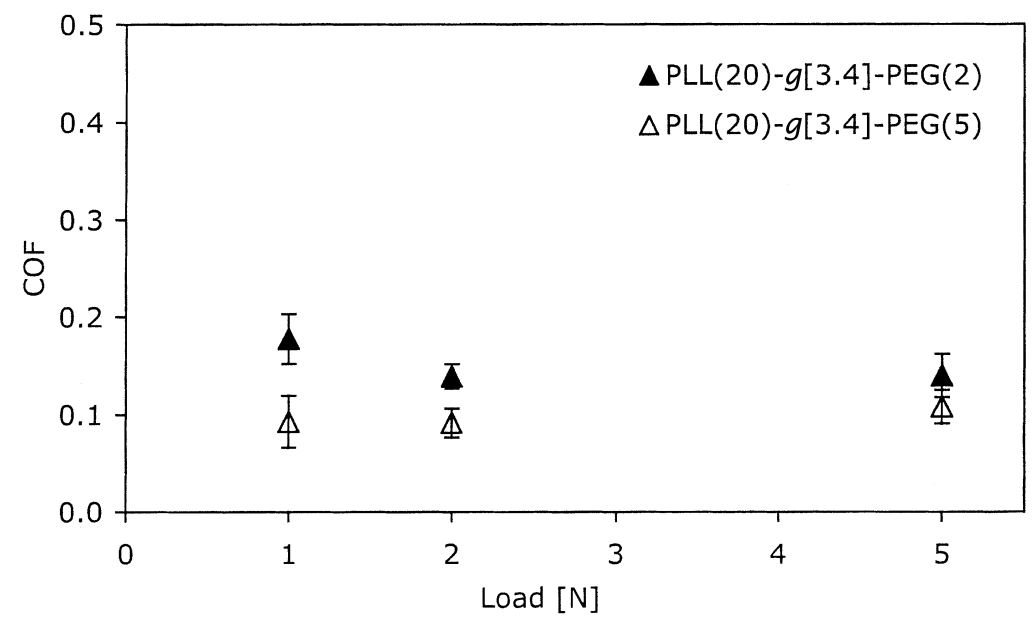

Figure 5. The influence of the molecular weight of the PEG side chains on the coefficient of friction was measured as a function of load by means of pin-on-disk tribometry. The test lubricants were aqueous buffer solutions containing either PLL(20)-g[3.4]-PEG(2) (black symbols) or PLL(20)-g[3.4]-PEG(5) (white symbols). Mean values of three experiments \pm standard deviation. Ball $=$ steel $(6 \mathrm{~mm}$ in diameter), disk $=$ glass, buffer solution $10 \mathrm{mM}$ HEPES ( $\mathrm{pH} 7.4$ ), polymer concentration $=0.25 \mathrm{mg} / \mathrm{ml}, \mathrm{load}=10 \mathrm{~N}, \mathrm{~T}=25^{\circ} \mathrm{C}$.

ultra-thin-film interferometry and MTM. Both film thickness and COF values for the two lower grafting ratios are very similar, although $g=2.2$ gives slightly lower friction, especially at low speed. However film thickness is significantly lower and COF higher for the highest grafting ratio, $g=5.7$. Figure 7 shows pin-ondisk measurements which reveal a similar COF dependence on grafting ratio in the decreasing order of $g=5.7>g=3.4>g=2.2$. In other words, the more PEG chains grafted on the PLL backbone, the lower the resulting $\mathrm{COF}$.

\subsubsection{Influence of the PLL backbone length}

The third structural parameter investigated was PLL backbone molecular weight. Two polymers having $20 \mathrm{kDa}$ and $350 \mathrm{kDa}$ PLL backbone molecular weight were compared: PLL(20)-g[3.4]-PEG(5) and PLL(350)$g$ [3.4]-PEG(5). The molecular weight of the PEG-sidechain $(5 \mathrm{kDa})$ and the PEG grafting ratio (3.4), were kept constant. Figure 8 shows the results of ultra-thinfilm interferometry and MTM. The higher molecular weight polymer shows both poorer film-forming properties and greater COF than the lower molecular weight

Film thickness:

COF:

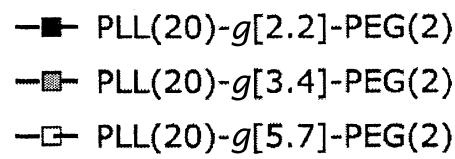

-20-PL(20)-g[2.2]-PEG(2)

-0 PLL(20)-g[3.4]-PEG(2)

$-Q-P L L(20)-g[5.7]-P E G(2)$

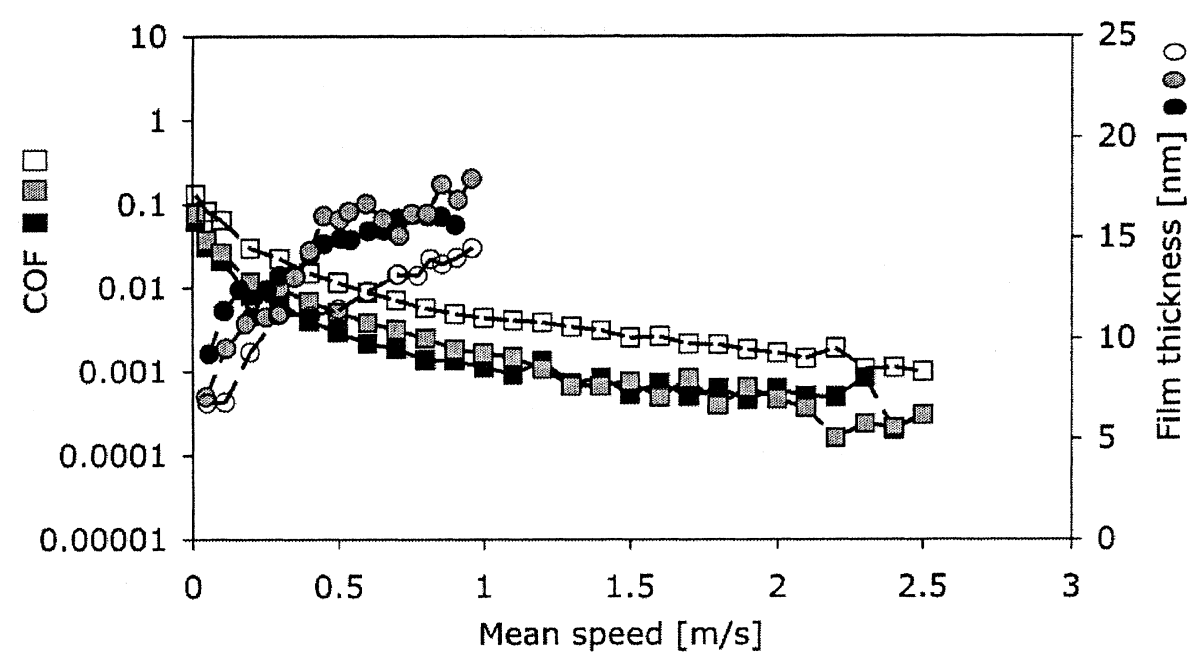

Figure 6. The influence of the Lys/PEG grafting ratio on the coefficient of friction (squares; y-axis on the left-hand-side) and lubricant film thickness (circles; y-axis on the right-hand-side) was measured as a function of speed by means of MTM and ultra-thin-film interferometry. The test lubricants were aqueous buffer solutions containing PLL(20)-g[2.2]-PEG(2) (black symbols), PLL(20)-g[3.4]-PEG(2) (grey symbols) or PLL(20)- $g$ [5.7]-PEG(2) (white symbols). The lines between data points serve as a guide for the eye. Ball $=$ stainless steel $(19 \mathrm{~mm}$ in diameter), substrate $=$ silica, buffer solution $=10 \mathrm{mM}$ HEPES $(\mathrm{pH} 7.4)$, polymer concentration $=0.25 \mathrm{mg} / \mathrm{ml}$, load $=10 \mathrm{~N}, \mathrm{~T}=25^{\circ} \mathrm{C}$. 


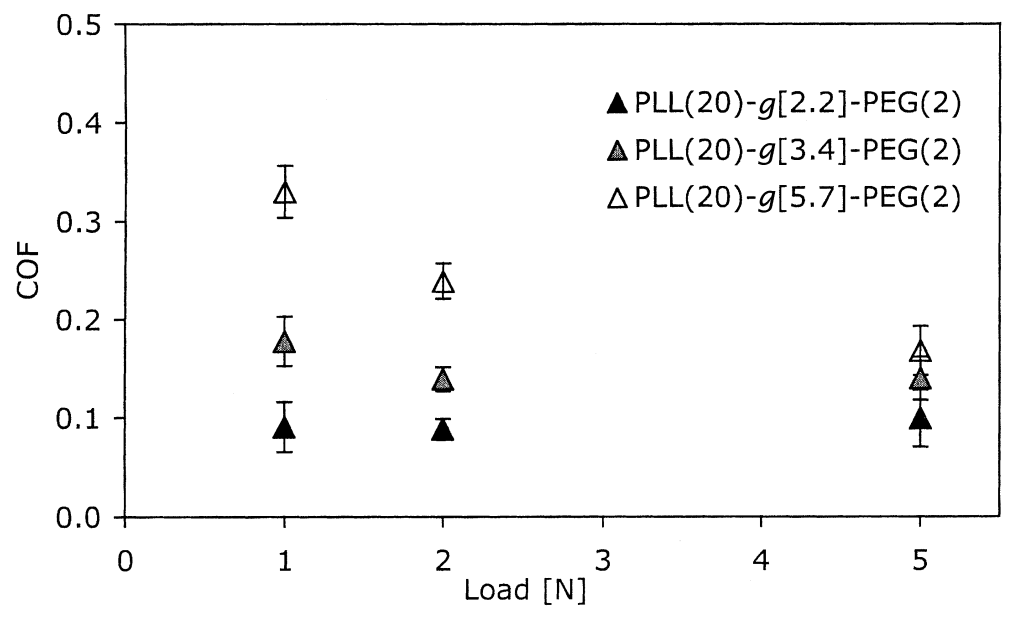

Figure 7. The influence of the Lys/PEG grafting ratio on the coefficient of friction was measured as a function of load by means of pin-on-disk tribometry. The test lubricants were aqueous buffer solutions containing PLL(20)-g[2.2]-PEG(2) (black triangles), PLL(20)-g[3.4]-PEG(2) (grey triangles) or PLL(20)-g[5.7]-PEG(2) (white triangles). Mean values of three experiments \pm standard deviation. Ball $=$ steel $(6 \mathrm{~mm}$ in diameter), disc $=$ glass, buffer solution $10 \mathrm{mM}$ HEPES $(\mathrm{pH} 7.4)$, polymer concentration $=0.25 \mathrm{mg} / \mathrm{ml}$, load $=10 \mathrm{~N}, \mathrm{~T}=25^{\circ} \mathrm{C}$.

one. The difference in film thickness is most marked at low speed and tends to be lost at high speed. However the difference in COF is very striking, with the lower molecular weight polymer giving friction at least an order of magnitude lower than the higher molecular weight one over almost the whole speed range. Interestingly, as shown in figure 9 , this difference between the two polymers was not seen in pin-on-disk tests.

\section{Discussion}

As shown above, the polymer PLL-g-PEG functions in aqueous solution as an effective boundary-lubricating additive and this effectiveness depend upon the polymer architecture. The focus of this discussion is to consider the mechanism of this boundary-lubricating performance and thus to determine how the polymer

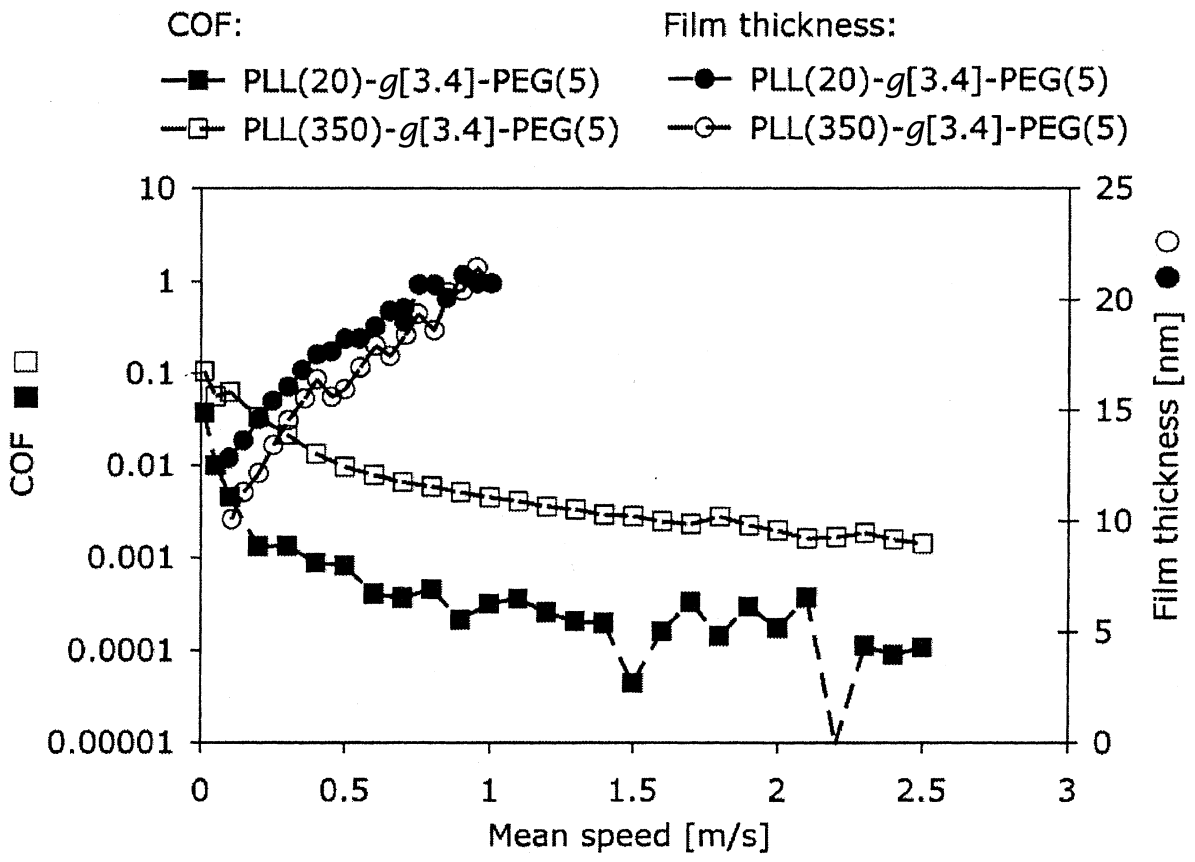

Figure 8. The influence of the molecular weight of the PLL backbone on the coefficient of friction (squares; $y$-axis on the left-hand-side) and lubricant film thickness (circles; y-axis on the right-hand-side) was measured as a function of speed by means of MTM and ultra-thin-film interferometry. The test lubricants were aqueous buffer solutions containing either PLL(350)- $g$ [3.4]-PEG(5) (white symbols) or PLL(20)-g[3.4]PEG(5) (black symbols). The lines between data points serve as a guide for the eye. Ball $=$ stainless steel $(19 \mathrm{~mm}$ in diameter $)$, substrate $=$ silica, buffer solution $=10 \mathrm{mM}$ HEPES $(\mathrm{pH} 7.4)$, polymer concentration $=0.25 \mathrm{mg} / \mathrm{ml}$, load $=10 \mathrm{~N}, \mathrm{~T}=25^{\circ} \mathrm{C}$. 


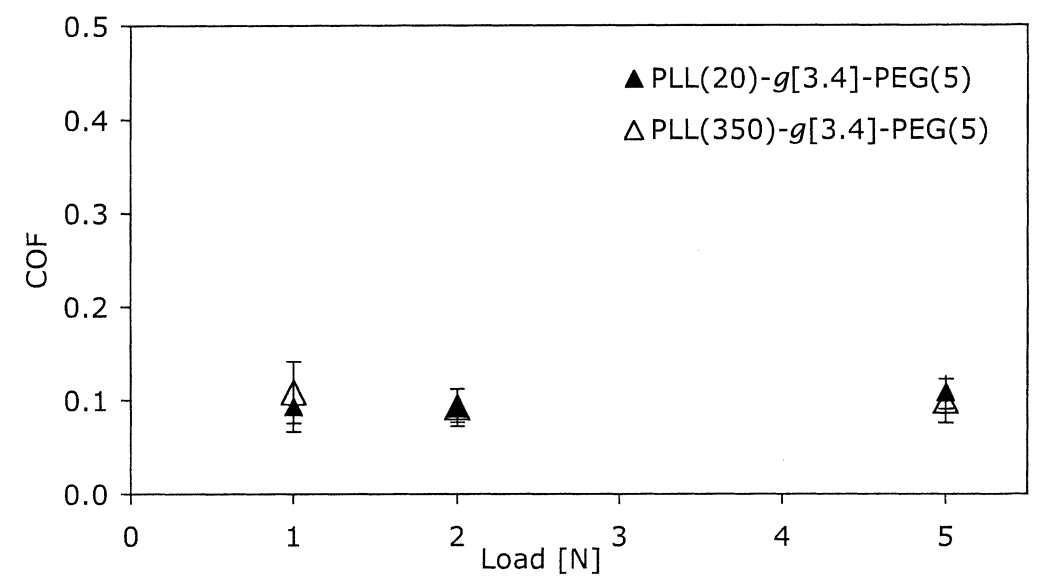

Figure 9. The influence of the molecular weight of the PLL backbone on the coefficient of friction was measured as a function of load by means of pin-on-disk tribometry. The test lubricants were aqueous buffer solutions containing PLL(350)-g[3.4]-PEG(5) (white triangles) and PLL(20)$g$ [3.4]-PEG(5) (black triangles). Mean values of three experiments \pm standard deviation. Ball $=$ steel $(6 \mathrm{~mm}$ in diameter $)$, disc $=$ glass, buffer solution $10 \mathrm{mM}$ HEPES (pH 7.4), polymer concentration $=0.25 \mathrm{mg} / \mathrm{ml}$, load $=10 \mathrm{~N}, \mathrm{~T}=25^{\circ} \mathrm{C}$.

molecules behave within a high-pressure contact to form a film and reduce friction.

\subsection{Lubrication regimes and lambda ratio}

According to elastohydrodynamic theory, in highpressure lubricated contacts, the motion of the surfaces entrains lubricant into the contact to form a film whose thickness is dependent on the mean speed, $U$, the dynamic viscosity, $\eta$, of the fluid in the contact inlet and the pressure viscosity coefficient, $\alpha$, of this fluid according to:

$$
h \propto(U \eta)^{\mathrm{a}}(\alpha)^{\mathrm{b}}
$$

where $\mathrm{a}$ and $\mathrm{b}$ are generally $0.6-0.7$ and 0.5 respectively [16].

This means that, at very low speeds, negligible fluid film is formed and the applied load is supported wholly by asperity-asperity contact between the solid surfaces. The friction is then determined by the shear strength properties of any thin adsorbed or reacted "boundary film" on these asperities. As the speed is raised, however, a fluid film gradually develops until, at high speeds, the surfaces are fully separated by a low-shear-strength, and thus low-friction, liquid lubricating film and the contact operates in full elastohydrodynamic lubrication.

One way of quantifying the extent of fluid film lubrication is in terms of the "lambda ratio", $\Lambda$, which is defined as the ratio between the film thickness $h$ and the out-of-contact, composite surface roughness of the sliding bodies [16]. In the current study, it was possible to calculate the lambda ratio directly, since the film thickness was measured. Therefore, since the contact conditions and tribo-pair were the same in both film thickness and MTM friction measurements, it was possible to determine the dependence of $\mathrm{COF}$ on lambda ratio for the fluids tested. This is shown in figure 10, where the COF (from MTM) is plotted against $\Lambda$ (calculated from $h$ measured by ultra-thin-film interferometry). Figure 10 illustrates two important features. Firstly, there is a rapid decrease in friction coefficient with increasing lambda ratio, which indicates that in all of the MTM measurements the contact is operating either in boundary or in mixed-film lubrication, depending on the experimental settings. In this regime, some of the load is supported by asperity contact and some by fluid film pressure, so that the friction arises from a combination of asperity and fluidfilm friction. As $\Lambda$ increases, the fluid-film component of the load support increases and thus the friction correspondingly decreases. A second feature of figure 10 is that the COF versus lambda ratio plots collapse onto one single line for all different PLL- $g$-PEG architectures, except for the single set synthesized from the high-molecular-weight PLL. This would be expected if the polymers were to have the same boundary friction at very low speed and the same fluid-film friction at higher speed. At intermediate speeds, friction would then depend solely on the polymer's contribution to fluid-film formation and thus on the $\Lambda$ ratio. The anomalous behavior of the high-molecular-weight PLLcontaining polymer will be discussed later.

\subsection{Film formation by HEPES and polymer solutions}

The tests on polymer-free HEPES showed negligible film formation below $1 \mathrm{~m} / \mathrm{s}$. This is primarily because HEPES, like pure water, has an extremely low pressure viscosity coefficient $\left(\alpha \sim 0.36 \mathrm{GPa}^{-1}\right.$ at $\left.55^{\circ} \mathrm{C}\right)$ compared to that of oil-based lubricants $\left(\alpha=10-20 \mathrm{GPa}^{-1}\right.$ at $55^{\circ} \mathrm{C}$ ) [17]. This means that, in accord with equation (1), it forms negligible fluid-film lubrication up to a mean speed of $1 \mathrm{~m} / \mathrm{s}$ [18]. The very high friction coefficient of 


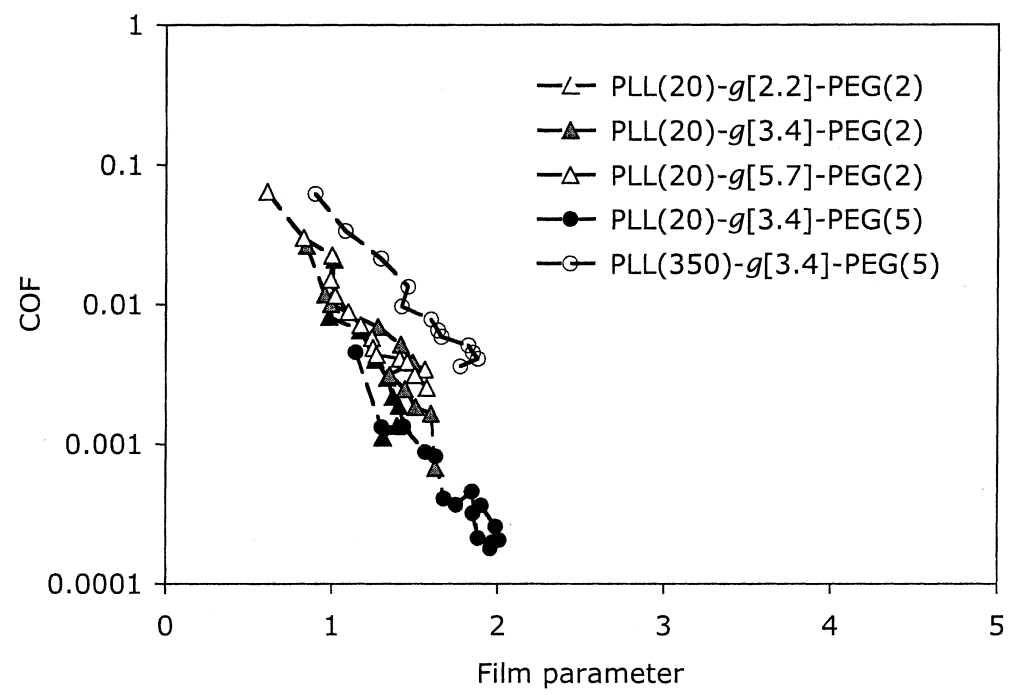

Figure 10. Friction coefficient as a function of the ratio of film thickness to surface roughness, $\Lambda$, showing regimes of lubrication [16]. The dimensionless film parameter $\Lambda$ is defined as the ratio between the film thickness $h_{c}$ and the composite surface roughness $R_{c}$ of the sliding bodies, $\Lambda=h_{c} / R_{c}$, where $R_{c}=\sqrt{R_{q, \text { ball }}^{2}+R_{q, \text { disk }}^{2}}$ and $R_{q \text {, ball }}$ and $R_{q \text {,disk }}$ are the RMS roughness of the ball (11 nm) and the disk ( $\left.2 \mathrm{~nm}\right)$ respectively. Thus $R_{c}=11.2 \mathrm{~nm}$.

HEPES at low speeds also indicates that the fluid possesses very poor boundary-film-forming properties.

In contrast to HEPES, the polymer solutions clearly show both a low-speed boundary-film formation (as evidenced by the pin-on-disk tests) and some degree of fluid-film entrainment. In conventional elastohydrodynamic (EHD) lubrication, as shown in equation (1), the film thickness should depend upon the mean speed raised to a constant exponent of between $a=0.6$ and 0.7 . Figure 11 shows a plot of $\log$ (film thickness) versus $\log$ (mean speed) for the polymer solution of PLL(20)$g$ [3.4]-PEG(2). The build-up of film thickness with speed is quite irregular but has gradient of $a \approx 0.3$. A similar gradient was found for all the polymers tested. This indicates that, although a film is clearly being entrained by the rolling/sliding motion of the surfaces, this entrainment does not conform with EHD theory. In figure 11 it can be seen that at a mean speed of above $0.8 \mathrm{~m} / \mathrm{s}$, the film thickness-speed curve appears to have become more regular, with a gradient approaching 0.6. This was also seen more clearly in previous work, which studied film thickness up to a speed of $3 \mathrm{~m} / \mathrm{s}$ [1] and is believed to represent the entrainment of bulk-water phase into the contact in a conventional EHD fashion. Previous work has shown that a significant EHD film starts to be formed by water at speeds above about $0.8 \mathrm{~m} / \mathrm{s}[18]$.

\subsection{Mechanism of film formation by $P L L-\mathrm{g}-P E G$}

From a combination of the film thickness and friction results it is possible to arrive at a coherent model for the molecular behavior of the PLL-g-PEG polymer in thin- film, high-pressure contact. In this model we consider that outside the contact region, the PLL chains are partially or wholly adsorbed on the surfaces with the hydrophilic PEG chains oriented outward into the buffer solution, building a brush-like structure that incorporates a large amount of coordinated water molecules $[12,19]$. Due to the strong hydrogen bonding interactions, the effective viscosity of the confined water will be increased several times. Within the contact zone, at zero or very slow speeds, the polymer brushes are effectively squashed down under load to a thickness of a few $\mathrm{nm}$. The friction coefficient under these conditions thus represents the shear strength of the squashed polymer and has a value of around 0.1 , typical of most adsorbed organic boundary-lubricating films. As the rolling/sliding speed is raised, however, the viscous film in the inlet, consisting of water confined in polymer, will begin to be entrained into the contact against the contact pressure to generate a quasi-elastohydrodynamic film. This will be promoted by an osmotic force that will tend to induce coordinated water molecules to move into the squashed polymer film $[3,20,21]$. As the speed is increased, the squashed PEG chains will respond to the entrained water by stretching and swelling, resulting in an increasing height of the brush layer with an associated increase of the normal force acting between the surfaces of the tribo-pair $[22,23]$. The thickness of this water/polymer film will continue to increase with entrainment speed until it reaches the uncompressed thickness of the brush layer upstream of the inlet and the polymer film can contribute no further to water entrainment. Thereafter, any further entrainment will be governed by the viscosity of bulk water and no further film growth occurs until the speed is sufficient 


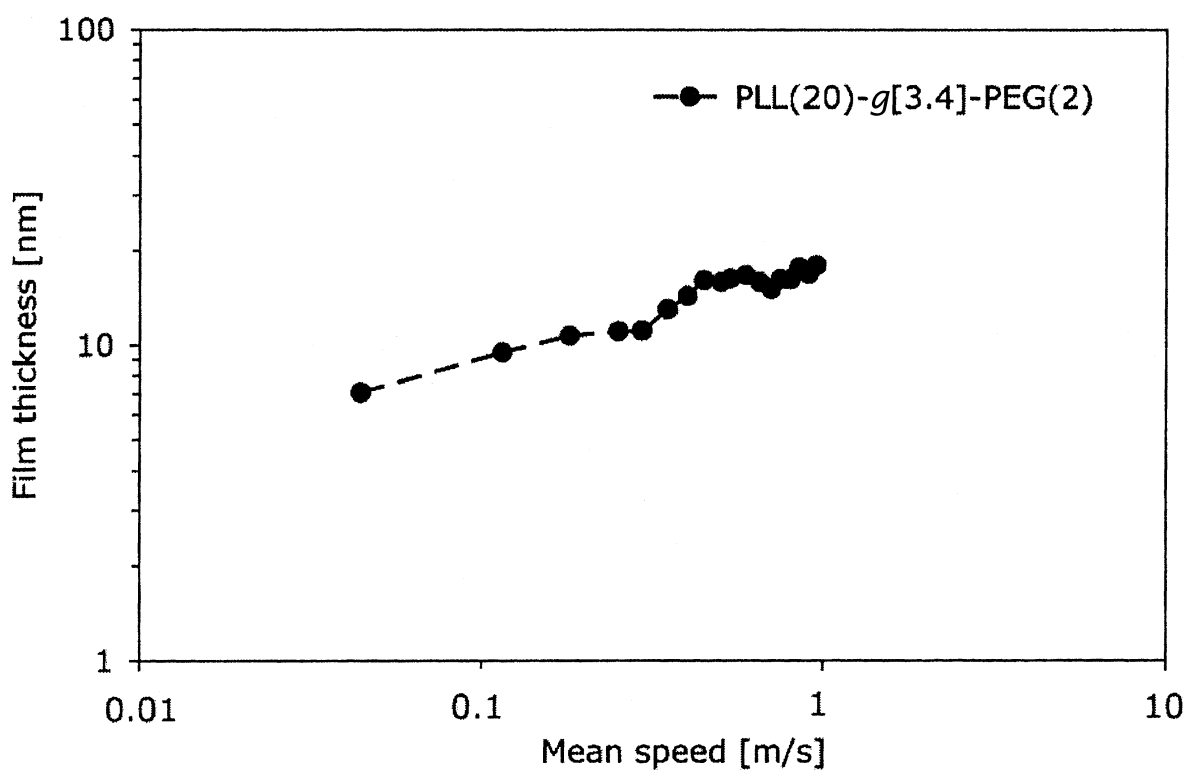

Figure 11. Log(film thickness) versus $\log$ (mean speed) for PLL(20)-g[3.4]-PEG(2)-containing HEPES buffer solution, measured by ultra-thinfilm interferometry. The lines between data points serve as a guide for the eye. Ball $=$ stainless steel $(19 \mathrm{~mm}$ in diameter $)$, substrate $=$ silica, buffer solution $=10 \mathrm{mM}$ HEPES $(\mathrm{pH} 7.4)$, polymer concentration $=0.25 \mathrm{mg} / \mathrm{ml}$, load $=10 \mathrm{~N}, \mathrm{~T}=25^{\circ} \mathrm{C}$.

for bulk water to lubricate via a conventional EHD mechanism. The leveling out of film thickness at around $20 \mathrm{~nm}$ noted in figure 11 (and figure 5 in [1]) thus represents the essentially full extension of the polymer PEG brushes.

\subsection{Influence of PLL-g-PEG architecture}

The architectural parameters investigated in this study were PEG side-chain length, Lys/PEG grafting ratio and backbone (PLL) length. First, the influence of the PEG chain length $\left(M_{w}=2\right.$ and $\left.5 \mathrm{kDa}\right)$ is discussed.

The radii of gyration $\left(R_{g}\right)$ of the unperturbed PEG chains in aqueous solution have been estimated in other work by static light-scattering measurements to be about 1.65 and $2.82 \mathrm{~nm}$ for PEG side chains of 2 and $5 \mathrm{kDa}$, respectively [11]. However, if the average spacing between two grafted PEG side chains is smaller than $2 R_{g}$, the coiled PEG side chains overlap, resulting in a stretching of the chains, resulting in a brush structure [19]. According to Alexander [24] and De Gennes [25], the brush height grows linearly with $\mathrm{N}$ under stretching conditions, while the nonstretched chain dimension $R_{g}$ only grows with $\mathrm{N}^{1 / 2}$. A stretching of the PEG side chains is thus expected to lead to a brush thickness that is much larger than $R_{g}$. As can be seen in figures 4 and 5 , an increase in the molecular weight of the PEG chain from 2 to $5 \mathrm{kDa}$ results in an increase in the interfacial film thickness by a factor of $\sim 1.2$ and in a significant reduction of the $\mathrm{COF}$ by a factor of $\sim 3.2$. It is suggested that the higher brush thickness associated with a higher water uptake, which results in an increase in $\Lambda$ ratio, is responsible for the superior lubrication properties of the polymer having higher-molecularweight PEG side chains.

The influence of the Lys/PEG grafting ratio on the lubrication properties was investigated by varying the grafting ratios $(2.2,3.4$, and 5.7). The results are shown in figure 6 and 7. By changing the Lys/PEG grafting ratio, both the number of PEG chains and the segmental charge of the polyelectrolyte molecule are changed, since the PEG chains are covalently bound via the $\mathrm{NH}_{2}$ terminated side chains of the poly(L-lysine) backbone, eliminating the charge contribution of the PEG-ylated amine groups. In other words, an increase in the Lys/ PEG grafting ratio leads to both a decreased number of PEG side chains per PLL-g-PEG molecule and an increase in the segmental charge on the PLL backbone. The latter leads to an additional decrease of the adsorbed PEG side-chain density on the negatively charged native oxide surface, since fewer polymer molecules are required to absorb in order to compensate the surface charge [26]. Hence, a reduction in the Lys/PEG grafting ratio should lead to an increasing PEG density on a negatively charged oxide surface. This has been confirmed by optical waveguide lightmode spectroscopy (OWLS) and time-of-flight secondary ion mass spectrometry (ToFSIMS) in a separate study [19]. As shown in figures 6 and 7, the COF for different Lys/PEG grafting ratios, measured by MTM and pin-on-disk tribometry, decreased in the order of $g=5.7>g=3.4>g=2.2$, which is consistent with the very reasonable conclusion that an increase in the PEG density on the interface of the tribo-pair results in an improvement of the lubrication properties of PLL-g-PEG.

The influence of the PLL backbone length was investigated by examining two polymers with very 
different PLL molecular weights (20 and $350 \mathrm{kDa})$. The influence of the PLL backbone length on the film thickness was found to be quite small, but its effect on MTM COF was considerable, with a significantly higher $\mathrm{COF}$ being found for the higher-molecular-weight PLL backbone. This difference is seen very clearly in figure 10 , where the high-molecular-weight PLL polymer lies on a markedly different COF versus $\Lambda$ line than the low molecular weight PLL polymers. This difference in COF must arise from the higher molecular weight PLL polymer having greater friction either in the very low speed, "squashed polymer boundary lubrication" condition or in the high speed, "extended brush fluid film" condition. However, the very low speed pin-on-disk test showed no influence of the molecular weight of the PLL backbone on the COF. Thus it seems likely that the larger COF for the high-molecular-weight PLL backbone polymer results from this polymer forming a more viscous water/polymer film in the fluid-film regime. The reason for this is not clear, but possibly the fluid film contains a significant proportion of unbound polylysine chain, which has a high resistance to shear within the contact.

\section{Conclusions}

The co-polymer poly (L-lysine)-g-poly(ethylene glycol) (PLL- $g$-PEG) has been investigated as a potential biomimetic boundary-lubrication additive for aqueous lubrication systems. In this work, the PLL-g-PEG polymers were found to provide both a boundary lubricating film at very slow sliding speeds and also to promote the entrainment of a fluid, separating film of up to $20 \mathrm{~nm}$ thickness at intermediate-to-high speeds. The development of a fluid film resulted in a very low friction coefficient. A model for the development of this fluid film has been proposed. Further, the influence of the copolymer architecture on the macroscopic tribological performance has been investigated. Both increasing the molecular weight of the PEG side chains and reducing the grafting ratio were found to result in an improvement in the lubricating properties of aqueous PLL- $g$ PEG solutions, in terms of increasing film thickness and friction reduction. Increasing the molecular weight of the PLL backbone resulted in an increase of the coefficient of friction at intermediate-to-high speeds but had little effect on film thickness.

The results of this study reveal the importance of the microscopic design of brush-like co-polyelectrolytes to control and optimize the lubrication capabilities of such polymer systems.

\section{Acknowledgments}

This work was financially supported by the Council of the Swiss Federal Institutes of Technology (ETH-Rat TopNano 21), the Swiss National Science Foundation NRP 47 and the US Air Force Office of Scientific Research (Contract No. F49620-02-1-0346). We are also grateful to Dr. Rowena Crockett of EMPA, Dübendorf, Switzerland, Monica Ratoi-Salagean of Imperial College, London, UK, and Stéphanie Pasche, BioInterface Group, LSST, Department of Materials, ETH-Zürich, for their valuable advice and assistance.

\section{References}

[1] S. Lee, M. Müller, J. Vörös, M. Ratoi-Salagean, H.A. Spikes and N.D. Spencer, Tribol. Lett., in press (2003).

[2] X. Yan, M.S. Lim, S. Pasche, S.M. De Paul, M. Textor, N.D. Spencer and S.S. Perry, submitted to Langmuir (2003).

[3] S.T. Milner, Science 251 (1991) 905.

[4] K. Kato, E. Uchida, E.-T. Kang, Y. Uyama and Y. Ikada, Progress in Polymer Science 28 (2003) 209.

[5] J.F. Joanny, Langmuir 8 (1992) 989.

[6] T.A. Witten, L. Leibler and P.A. Pincus, Macromolecules 23 (1990) 824.

[7] J. Klein, E. Kumacheva, D. Mahalu, D. Perahia and L.J. Fetters, Nature 370 (1994) 634.

[8] U. Raviv, R. Tadmor and J. Klein, J. Phys. Chem. B 105 (2001) 8125 .

[9] T. Bouhacina, J.P. Aime, S. Gauthier, D. Michel and V. Heroguez, Phys. Rev. B 56 (1997) 7694

[10] G.S. Grest, Current Opinion in Colloid \& Interface Science 2 (1997) 271.

[11] G.S. Grest, Adv. Polymer Sci. 138 (1999) 149.

[12] G.L. Kenausis, J. Vörös, D.L. Elbert, N.P. Huang, R. Hofer, L. Ruiz-Taylor, M. Textor, J.A. Hubbell and N.D. Spencer, J. Phys. Chem. B 104 (2000) 3298.

[13] N.P. Huang, R. Michel, J. Vörös, M. Textor, R. Hofer, A. Rossi, D.L. Elbert, J.A. Hubbell and N.D. Spencer, Langmuir 17 (2001) 489.

[14] G.J. Johnston, R. Wayte and H.A. Spikes, Tribol. Trans. 34 (1991) 187

[15] M. Smeeth and H. Spikes, 22nd Leeds/Lyon Symposium on Tribology, The Third Body Concept Proc. (1996) p. 695.

[16] B.J. Hamrock, Fundamentals of Fluid Film Lubrication (McGrawHill, 1994).

[17] P. Vergne, M. Kamel and M. Querry, ASME J. Tribol. 119 (1997) 250.

[18] M. Ratoi and H.A. Spikes, Tribol. Trans. 42 (1999) 479.

[19] S. Pasche, S.M. De Paul, J. Vörös, N.D. Spencer and M. Textor, submitted to Langmuir (2003).

[20] J.L. Barrat, Macromolecules 25 (1992) 832

[21] Y. Rabin and S. Alexander, Europhysics Lett. 13 (1990) 49.

[22] J. Klein, E. Kumacheva, D. Perahia, D. Mahalu and S. Warburg, Faraday Disc. (1994) 173.

[23] B. Bhushan, J.N. Israelachvili and U. Landman, Nature 374 (1995) 607

[24] S. Alexander, Le Journal de Physique 38 (1977) 977.

[25] P.G. De Gennes, Macromolecules 13 (1980) 1069.

[26] N.G. Hoogeveen, M.A.C. Stuart and G.J. Fleer, J. Coll. Inter. Sci. 182 (1996) 133. 